\author{
A MESH - PARAMETER - CONTINUATION \\ METHOD \\ S.J. Polak \\ A. Wachters \\ Th. Beelen \\ N.V. Philips' Gloeilampenfabrieken \\ Eindhoven, The Netherlands \\ P.W. Hemker \\ Mathematisch Centrum \\ Amsterdam, The Netherlands
}

I. INTRODUCTION

The most important aspect in the construction of program packages for P.D.E. problems [1-3] is Robustness. Other criteria (in order of importance) are user-friendliness and speed. This paper describes our present efforts to construct Robust algorithms for the solution of a class of nonlinear P.D.E. problems.

The algorithms are only considered with respect to a special class of problems (section 2) but extensions to other problems are obvious. The predictor - corrector - continuation (PCC) method [4] is one of the most Robust algorithms known to us for the solution of nonlinear equations.

Combinations of the PCC method and spatial discretizations are therefore obvious choices when constructing Robust algorithms for P.D.E. problems [5]. Two different combinations are considered in this paper. First a continuation parameter $t$ is introduced in the continuous problem to solve the 
problem on a single given mesh (section 4). Secondly a PCC method is used for the solution of the residual problem arising from a two mesh algorithm (section 5). In section 6 we consider the simultaneous use of both algorithms. In section 7 a practical implementation of this combination is described.

\section{SEMICONDUCTOR PROBLEMS}

The equations involved in the analysis of semiconductor problems are discussed in detail in [6], [7] and [3]. Here we only consider the Poisson problem for the case of negligible currents. In [3] this problem is treated for physically realistic 2-D composite regions.

In this paper we use only a $1-D$ simplified problem as an example. The equation has the form

$$
-\Delta u=H(u),
$$

where

$$
H(u)=a\left(\exp b\left(c_{1}-u\right)-\exp b\left(u-c_{2}\right)\right)+D(x),
$$

with

$$
\begin{aligned}
& \mathrm{x} \in[20 \mu, 100 \mu], \mu=10 * *-6, \mathrm{u}(20 \mu)=0, \mathrm{u}(100 \mu)=700, \\
& \mathrm{a}=10 * * 13, \mathrm{~b}=40, \mathrm{c}_{1}=0, \mathrm{c}_{2}=700 \text { and } \\
& \mathrm{D}(\mathrm{x})=-\mathrm{a} \text { if } \mathrm{x}<50 \mu, \mathrm{D}(\mathrm{x})=\mathrm{a} \text { if } \mathrm{x}>50 \mu .
\end{aligned}
$$

The problem will also be denoted by $L(u)=0$. There exists a unique bounded solution for this problem. This is also the case for the more general problem treated in [3]. To understand this, a theorem from [8] is used. The basic property there is monotony. Our operator is monotonic because $-\Delta$ is coercive and $\mathrm{H}_{\mathrm{u}}(\mathrm{u})$ is negative definite.

The upper and lower bound of the solution of the problem are $c_{1}-\exp \left(b\left(c_{1}-c_{2}\right)\right) / b$ and $c_{2}+\exp \left(b\left(c_{1}-c_{2}\right)\right) / b$ respectively, as can be easily shown by techniques similar to those used for the proof of a maximum principle [10]. These bounds imply that, within the range of floating point representable numbers, the solution of (1) is a monotonous function. In the general case, a priori lower and upper bounds for the solution are 
known on physical grounds. Therefore, $a, c_{1}$ and $c_{2}$ are always such that, within rounding errors, $c_{1}-u, u-c_{2} \leq 0$; hence, in (1) the function $\exp (x)$ may be replaced by $1+x$ for $x \geq 0$. This does not change the solution of the problem and it eliminates the problems caused by the large exponential factor $b$.

III. THE PCC METHOD

The PCC method is described in [3] and [4]. Its use for semiconductor potential problems is discussed in [3]. The algorithm is summarized here and a new step strategy is presented.

Suppose we want to solve the equation

$$
F(u)=0
$$

Then the continuation parameter $t$ is introduced giving

$$
\widetilde{F}(u(t), t)=0,0 \leq t \leq 1 \text {, }
$$

with $\tilde{F}(u(1), 1)=F(u(1))=0$ and the initial solution $u(0)$ is known.

Differentiation with respect to $t$ gives

$$
\tilde{F}_{t}+\tilde{F}_{u} u_{t}=0
$$

The solution of (3) is approximated at $0=t_{0}<\ldots<t_{n}<\ldots<t_{n}=1$, the steplength is denoted by $\tau_{n}=t_{n}-t_{n-1}$.

With equation (4) forward Euler is used to compute the prediction $\vec{u}_{\left(t_{n}\right.}$ ) and Newton's method is applied to (3) to find the solution $u\left(t_{n}\right)$.

Because we were not satisfied with the existing step strategies we devised a new one, which proved to be better both in the 1-D model and in the realistic 2-D problems.

Set $e_{i, n}=\left\|u_{i}\left(t_{n}\right)-u_{i-1}\left(t_{n}\right)\right\|, i=1, \ldots, J+1, u_{0}\left(t_{n}\right)=\bar{u}\left(t_{n}\right)$, define $\alpha_{i, n}=e_{i+1, n} / e_{i, n}^{2}$. Then the mean quadratic convergence factor is defined by $\bar{\alpha}_{n}=\sqrt[i+1]{\mathrm{P}}$, with $\mathrm{P}=\Pi_{i=1}^{\mathrm{J}} \alpha_{i, \mathrm{n}^{\prime}}^{\mathrm{s}_{i}} \mathrm{~s}_{i}=2^{\mathrm{J}-i}$ and $\mathrm{m}=2^{\mathrm{J}-1}-1$ where $u_{\mathrm{J}+1}\left(t_{\mathrm{n}}\right)$ is the final value of the Newton iterations.

Suppose we want to accept $u_{I}\left(t_{n+1}\right)$ if $e_{I, n}<\delta$ for some chosen tolerance $\delta$ and number of iterations $I$. Suppose $u_{0}\left(t_{n+1}\right)=u\left(t_{n+1}\right)$ is calculated with an integration method, e.g. forward Euler. Then 


$$
e_{0, n+1} \leq\left\|u_{0}\left(t_{n+1}\right)-u_{I}\left(t_{n+1}\right)\right\|+\alpha\left\|u_{0}\left(t_{n+1}\right)-u_{I}\left(t_{n+1}\right)\right\|^{2},
$$

where $\alpha$ is the convergence factor of Newton at $t$. Now we use $\bar{\alpha}_{n}$ as approximation for $\alpha$. Thus

$$
e_{0, n+1} \leq \varepsilon(\tau)+\bar{\alpha}_{n} \varepsilon(\tau)^{2}
$$

where $\varepsilon(\tau)$ is the truncation error of the integration method and from

$$
e_{I-1, n+1} \leq \bar{\alpha}_{n}^{2 I-1} e_{0, n+1}^{2^{I}} \leq \bar{\alpha}_{n} 2^{I}-1\left(\varepsilon(\tau)+\bar{\alpha}_{n}\left(\varepsilon(\tau)^{2}\right)^{2^{I}}\right) \leq \delta,
$$

$\tau$ can be calculated. The step changes are limited by $\tau_{n-1} / 2 \leq \tau_{n} \leq 3 \tau_{n-1}$. we start with trying $\tau_{1}=1$. If $\tau_{1}=1$ fails then $\tau_{1}=\tau_{1} / 5$ etc. until $\tau_{1}$ is accepted. A t-step is rejected if

$$
F\left(u_{i+1}\right) / F\left(u_{i}\right)>.9 \text { and } F\left(u_{i+2}\right) / F\left(u_{i+1}\right)>.9
$$

for some $i \geq 1$. Then the step is divided by 2 .

$$
\text { IV. PARAMETER - CONTINUATION (PC) }
$$

The parameter $t$ is introduced in (1) by replacing $H(u)$ by $t \times H(u)$. Thus at $t=0$ the problem has a smooth solution (linear) and at $t=1$ the resulting singular perturbation problem has a solution with an interior boundary layer. To approximate the solution, the problem is discretised a FEM on a uniform mesh. The resulting equations are solved with the PCC method. For 3 different meshes the number of t-steps, rejected steps and Newton iterations are shown in table I.

TABLE I.

\begin{tabular}{|l|c|c|c|}
\hline number of mesh intervals & 8 & 32 & 128 \\
\hline Newton iterations & 9 & 125 & 418 \\
\hline t-steps accepted (rejected) & $1(0)$ & $27(2)$ & $92(5)$ \\
\hline
\end{tabular}




\section{MESH - CONTINUATION}

Let $G_{i}$ and $G_{i+1}$ be two meshes, $G_{i}$ being a submesh of $G_{i+1}$. The operator $I_{i+1}^{i}$ interpolates a function defined on $G_{i}$, yielding a function defined on $G_{i+1}$. The discretization of $L(u)$ on $G_{j}$ is indicated by $L_{j}(u), j=i, i+1$. Suppose $L_{i}\left(u_{i}\right)=0$, then we calculate $u_{i+1}$, defined by $L_{i+1}\left(u_{i+1}\right)=0$, from

$$
(1-t) L_{i+1}\left(I_{i+1}^{i} u_{i}\right)-I_{i+1}\left(\tilde{u}_{i+1}(t)\right)=0 \text {, }
$$

with $\tilde{u}_{i+1}(0)=I_{i+1}^{i} u_{i}$ and $u_{i+1}=\tilde{u}_{i+1}(1)$. Again we use the PCC algorithm to solve this problem.

Table II shows the number of Newton iterations needed to proceed from the 8 to the 32 interval mesh, from the 32 to the 128 interval mesh and from the 8 to the 128 interval mesh.

The interpolation operator is defined by locally solving the problem with coarse grid solutions as boundary values and, again, a PCC method.

TABLE II. Number of Newton iterations

\begin{tabular}{|c|c|c|c|}
\hline number of mesh intervals & 8 & 32 & 128 \\
\hline parameter continuation (PC) & 9 & 125 & 418 \\
\hline $\begin{array}{c}\text { PC at coarse mesh }+ \\
\text { mesh continuation (MPC) }\end{array}$ & $9 \frac{1}{\mid}+3 \frac{1}{\mid}+6$ \\
\hline
\end{tabular}

If the solutions on the different meshes are sufficiently close, no continuation method is required to solve $L_{i+1}\left(u_{i+1}\right)=0$, i.e. the mesh continuation is simply Newton with $I_{i+1}^{i} u_{i}$ as the initial estimate. However, if $I_{i+1}^{i} u_{i}$ is not sufficiently close (and for most realistic problems we do not have this a priori knowledge), then the mesh-continuation improves the robustness essentially. 
VI. MESH - PARAMETER - CONTINUATION (MPC)

The algorithms from sections 5 and 6 may be combined to give a Robust and fast algorithm. The basic idea, of course, is to solve small sets of equations first, using parameter continuation on the coarse mesh. Then the solution is calculated on a sufficiently fine mesh using mesh-continuation. In the following section we present a practical implementation of this combination.

If we take the coarse mesh too coarse, the solution may be a very bad approximation of fine mesh solution. Then the work for the MPC algorithm may be more than the work necessary for the simple PC algorithm on the fine mesh. Therefore, in future we shall try to construct algorithms which use a sequence of meshes and optimize the path followed in the two parameter embedding provided by the combination of mesh and parameter continuation. of course, in this context [5] and [9] should be mentioned. However, in those papers, Robustness is not the primary concern.

VII. 2-D MESHES

The 2-D meshes used e.g. in the program packages MAGGY2 [1] and SEMMY2 [3] are very suited for the algorithm as described in section 6 . The meshes used in these packages are discussed in [1]. The meshes consist of quadrilaterals and triangles. They may be considered as a distortion of a rectangular mesh. Coinciding points yield triangles. These meshes are used for $\mathrm{FE}$ discretizations and they allow an easy description in the problem oriented language as can be seen in Figure 1.

We see that a coarse and a fine mesh are given at the same time by the user of the package [1], the fine mesh being constructed from the coarse mesh by linear interpolation on the coordinate space. Realistic problems with complicated geometries, solved with such meshes, can be found in [1] and $[2]$. 

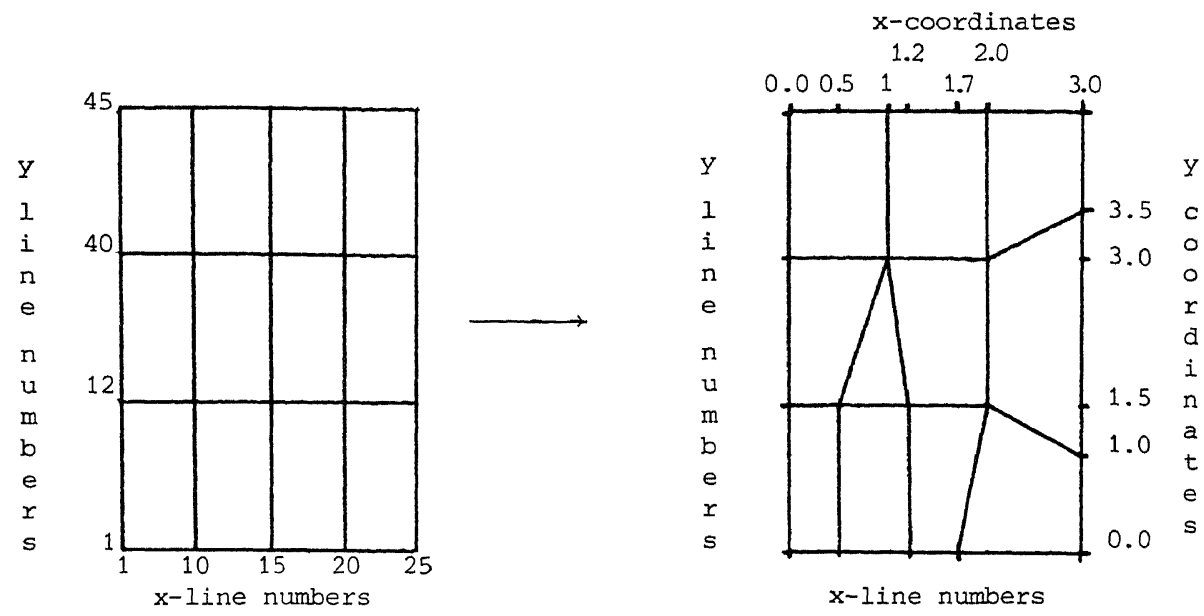

FIGURE 1. The mesh is specified in the SEMMY2 input language by:

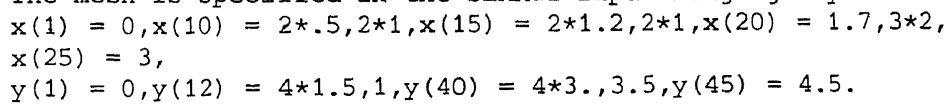

REFERENCES

1. Polak, S.J., de Beer, A., Wachters, A. and van Welij, J.S. (1980). MAGGY2, A programpackage for two dimensional magnetostatic problems.

Int. J. for Num. Meth. in Eng. Vol. 15, 113-127.

2. Polak, S.J., Schrooten, J. and Barneveld Binkhuysen, C. (1978). TEDDY2, A programpackage for parabolic composite region problems. ACM TOMS vol. 4, no. 3, 209-227.

3. Polak, S.J., Wachters, A., Vaes, H.J.M., de Beer, A. and den Heyer, C. (1979). A continuation method for the calculation of electrostatic potentials in semiconducturs. In: Proc. of the NASECODE I Conference, Trinity College, Boole Press, Dublin.

4. Rheinboldt, W.C. (1976). In: "Mathematics of FEM and applications" J. Whiteman, ed.. Academic Press, London, pp. 465-482.

5. Babuska, I. and Rheinboldt, W.C., Reliable error estimation and mesh adaption for the FEM. Tech. note BM910, Univ. of Maryland, april 1979.

6. Slotboom, J.W. (1977). Analysis of bipolar transistors, Thesis,

Technische Hogeschool, Eindhoven. 
7. Mock, M.S. (1972). On equations describing steady-state carrier distributions in a semiconductor device. Comm. Pure and Appl. Math. Vol.25, $781-792$.

8. Ciarlet, P.C., Schultz, M.H. and Varga, R.S. (1969). Numer. Math. 13, 51-77.

9. Zave, P. and Rheinboldt W.C. (1979). Design of an adaptive parallel finite element system. ACM-TOMS, Vol. 5, no. 1, 1-17.

10. Protter, H.H. and Weinberger, H.F. (1967). Maximum principles in differential equations. Prentice Hall. 